\title{
On quadratic twists of elliptic curves and some applications of a refined version of Yu's formula
}

\author{
Derong Qiu * \\ (School of Mathematical Sciences, Capital Normal University, \\ Beijing 100048, P.R.China )
}

\begin{abstract}
In this paper, we study some cohomology groups and quadratic twists of elliptic curves, and apply Tate local duality and the results of KramerTunnell on local norm cokernel to give a refined version of Yu's formula in the case of elliptic curves. Then, by using this refinement formula, we obtain explicit orders of Shafarevich-Tate groups of some elliptic curves in quadratic number fields, including a few unconditional cases.
\end{abstract}

Keywords: Elliptic curve, quadratic twist, cohomology group, ShafarevichTate group, Heegner point.

2010 Mathematics Subject Classification: $11 \mathrm{G} 05$ (primary), 14H52, 14G05, 14G10 (Secondary).

\section{Introduction}

Let $K / F$ be a finite Galois extension of number fields with Galois group $G, A$ an abelian variety defined over $F$, and let $\mathbf{I I I}(A / F), \mathbf{I I I}(A / K)$ denote the ShafarevichTate groups of $A$ over $F$ and $K$, respectively. In $[Y]$, under the assumption that these groups are finite, Yu computed the value $\sharp \mathbf{I I I}(A / K)^{G} / \sharp \mathrm{III}(A / F)$. In particular, when

\footnotetext{
* E-mail: derong@mail.cnu.edu.cn, derongqiu@gmail.com
} 
$K$ is a quadratic extension of $F, \mathrm{Yu}$ derived a formula relating $\amalg(A / F), \amalg(A / K)$ and $\amalg\left(A^{\chi} / F\right)$ as follows, where $A^{\chi}$ is the twist of $A$ by the non-trivial character $\chi$ of $G$.

Yu's formula (see [Y], Main Theorem, p.212) Assume that the ShafarevichTate groups are finite. Let $A^{\prime}$ be the dual abelian variety of $A$. Then

$$
\frac{\sharp \amalg(A / F) \cdot \sharp \amalg\left(A^{\chi} / F\right)}{\sharp \amalg(A / K)}=\frac{\sharp \widehat{H}^{0}\left(G, A^{\prime}(K)\right) \cdot \sharp H^{1}(G, A(K))}{\prod_{v \in M_{F}} \sharp H^{1}\left(G_{v_{K}}, A\left(K_{v_{K}}\right)\right)},
$$

where $v_{K}$ is the fixed place of $K$ lying above $v$ for each $v \in M_{F}$.

A further question is to determine these quantities of the right-hand side of this equality, which seems not too easy to practically calculate in general.

In this paper, for the case of elliptic curves, we refine the above Yu's formula in an elementary and simpler form. In fact, by studying some cohomology groups of quadratic twists of elliptic curves, we can relate the order of $H^{1}(G, A(K))$ with the groups of rational points of $A$ and its twist (see Theorem 1.5 in the following Section 1), then by applying Tate local duality (see [Ta1]), and the results of Kramer and Tunnell on local norm cokernel (see Prop.1 5 in $[\mathrm{Kr}]$ and Thm.7.6 in[KT]), we can in some extent calculate the order of local cohomology groups $H^{1}\left(G_{v_{K}}, A\left(K_{v_{K}}\right)\right)$. Our refined version of Yu's formula in the case of elliptic curves is as follows

A refined version of Yu's formula (see Theorem 2.1 in the following). Assume that the Shafarevich-Tate groups $\amalg(E / F), \amalg\left(E_{D} / F\right)$ and $\amalg(E / K)$ are finite. Then

$$
\frac{\sharp \amalg(A / F) \cdot \sharp \amalg\left(A_{D} / F\right)}{\sharp \amalg(A / K)}=2^{r_{D, F}-r_{F}-\delta(A, F, K)} \cdot(A(F): N(A(K)))^{2},
$$

where the value $\delta(A, F, K)$ can often be explicitly calculated (see Theorem 2.1 in 
the following Section 2 for a precise meaning of $\delta(A, F, K))$.

One of our motivation here is, Yu's formula makes it possible to determine the Shafarevich-Tate group of $A$ over the quadratic extension $K$, when these groups of $A$ and its twist over $F$ are known. One of the uses of our refined version of this formula is that the values $\delta(A, F, K)$ can be usually directly calculated (e.g., see Lemma 3.2 and the proof of Thm.4.1 in the following). As for applications, by this refined version of Yu's formula, and using some known results of ShafarevichTate groups of several family of elliptic curves over $\mathbb{Q}$, we obtain explicit orders of their Shafarevich-Tate groups over some quadratic number fields, including a few unconditional cases (see Theorems 3.3, 4.1 and Corollary 3.4 in the following).

\section{Quadratic twists and cohomology groups}

Let $F, K$ be number fields with $K=F(\sqrt{D})$ a quadratic extension of $F$ for some $D \in F^{*} \backslash F^{*^{2}}$. Let $G=\operatorname{Gal}(K / F)=<\sigma>$ be its Galois group with a generator $\sigma$. Let $E: y^{2}=x^{3}+a x+b$ be an elliptic curve defined over $F$. Its quadratic $D$-twist is given by $E_{D}: y^{2}=x^{3}+a D^{2} x+b D^{3}$. By the Mordell-Weil Theorem (see [Si1,Thm.6.7,p.239]), the group $E(F)$ of $F$-rational points of $E$ is a finitely generated abelian group, so are the groups $E_{D}(F)$ and $E(K)$. For simplicity, in the following, we denote $r_{F}=\operatorname{rank} E(F), r_{D, F}=\operatorname{rank} E_{D}(F)$ and $r_{K}=\operatorname{rank} E(K)$. Let IIII $(E / F)$, IIII $\left(E_{D} / F\right)$ and III $(E / K)$ be the Shafarevich-Tate groups of $E$ over $F, E_{D}$ over $F$ and $E$ over $K$ respectively (see [Si1, p.332] for the definition).

Since $E$ and $E_{D}$ are $K$-isomorphic as given by

$$
\phi_{D}: E_{D} \longrightarrow E, \quad(x, y) \longmapsto\left(\frac{x}{(\sqrt{D})^{2}}, \frac{y}{(\sqrt{D})^{3}}\right)
$$


we have $E(K) \cong E_{D}(K), \amalg\left(E_{D} / K\right) \cong \amalg(E / K)$ and $r_{F}+r_{D, F}=r_{K}$ (see [ABF, p.5], [RS, Lemma 2.1]). Denote

$$
R_{D}(F)=\phi_{D}\left(E_{D}(F)\right)=\left\{\left(\frac{x}{D}, \frac{y}{(\sqrt{D})^{3}}\right):(x, y) \in E_{D}(F)\right\} \cup\{O\} \subset E(K) .
$$

Obviously, $R_{D}(F)$ is a subgroup of $E(K)$, and $R_{D}(F) \cong E_{D}(F)$ as abstract groups.

Throughout this paper, for a set $S$, we denote its cardinal by $\sharp S$. For arbitrary abelian group $A$ and positive integer $m$, we denote $m A=\{m a: a \in A\}$ and $A[m]=\{a \in A: m a=0\}$. If $A$ is a $G$-module, then one has the following Tate cohomology groups : $\widehat{\mathrm{H}}^{n}(G, A)=\mathrm{H}^{n}(G, A)$ if $n \geq 1 ; \widehat{\mathrm{H}}^{0}(G, A)=A^{G} /(1+\sigma) A$. For the basic facts of cohomology groups $\mathrm{H}^{n}(G, A)(0 \leq n \in \mathbb{Z})$ and Tate cohomology groups $\widehat{\mathrm{H}}^{m}(G, A)(m \in \mathbb{Z})$ of $G$-module $A$, see [Se, Chapt.VIII] and [AW, $\left.\S 6\right]$.

Lemma 1.1. $R_{D}(F)=\{P \in E(K): \sigma(P)=-P\}$.

The two maps $\varphi_{1}: E(K) \longrightarrow E(K), \quad P \longmapsto P+\sigma P \quad(\forall P \in E(K))$ and $\varphi_{2}: E(K) \longrightarrow E(K), \quad P \longmapsto P-\sigma P \quad(\forall P \in E(K))$ are endomorphisms of abelian group $E(K)$ with kernels $\operatorname{ker} \varphi_{1}=R_{D}(F)$ and $\operatorname{ker} \varphi_{2}=E(F)$ respectively. We denote $N(E(K))=\operatorname{im} \varphi_{1}$, the images of $\varphi_{1}$; and $T_{D}(F)=\operatorname{im} \varphi_{2}$, the images of $\varphi_{2}$. Obviously $N(E(K)), T_{D}(F)$ and $R_{D}(F)$ are finitely generated abelian groups because they are subgroups of $E(K)$. We have

$$
2 E(F) \subset N(E(K)) \subset E(F), \quad 2 R_{D}(F) \subset T_{D}(F) \subset R_{D}(F) .
$$

Moreover, by the former discussion and the exact sequences of abelian groups $O \rightarrow$ $R_{D}(F) \rightarrow E(K) \rightarrow N(E(K)) \rightarrow O$ and $O \rightarrow E(F) \rightarrow E(K) \rightarrow T_{D}(F) \longrightarrow O$, we have $\operatorname{rank} E(K)=\operatorname{rank} R_{D}(F)+\operatorname{rank} N(E(K))=\operatorname{rank} E(F)+\operatorname{rank} T_{D}(F)$, $\operatorname{rank} T_{D}(F)=\operatorname{rank} E_{D}(F)=\operatorname{rank} R_{D}(F), \quad \operatorname{rank} N(E(K))=\operatorname{rank} E(F)$. 
In particular, the quotient groups $E(F) / N(E(K))$ and $R_{D}(F) / T_{D}(F)$ are finite abelian groups.

\section{Lemma 1.2 .}

(1) $R_{D}(F)[2]=R_{D}(F) \cap E(F)=E(F)[2]$.

(2) $T_{D}(F)[2]=T_{D}(F) \cap E(F)=T_{D}(F)^{G} \subset R_{D}(F)^{G}=E(F)[2]$.

(3) The inverse images of $2 R_{D}(F), 2 E(F)$ under $\varphi_{2}, \varphi_{1}$ respectively are given by $\varphi_{2}^{-1}\left(2 R_{D}(F)\right)=E(F)+R_{D}(F)=\varphi_{1}^{-1}(2 E(F))$.

\section{Lemma 1.3.}

$\left(E(K): E(F)+R_{D}(F)\right)=\left(T_{D}(F): 2 R_{D}(F)\right)=(N(E(K)): 2 E(F))$.

The proofs of Lemmas 1.1 1.3 are straightforward.

For the $G$-modules $E(K), E(F), R_{D}(F), T_{D}(F)$ and $N(E(K))$, we have the following results about their corresponding cohomology groups:

Proposition 1.4. $\mathrm{H}^{1}(G, E(K))=R_{D}(F) / T_{D}(F) ; \quad \mathrm{H}^{1}(G, E(F))=E(F)[2] ;$

$\mathrm{H}^{1}\left(G, R_{D}(F)\right)=R_{D}(F) / 2 R_{D}(F) ; \quad \mathrm{H}^{1}\left(G, T_{D}(F)\right)=T_{D}(F) / 2 T_{D}(F) ;$

$\mathrm{H}^{1}(G, N(E(K)))=N(E(K))[2]$.

Proof. Since $G$ is cyclic, by the explicit formulae of cohomology of finite cyclic groups (See [Se], pp.133, 128 for the details), we have

$\mathrm{H}^{1}(G, E(K))=\operatorname{ker} \varphi_{1} / \operatorname{im} \varphi_{2}=R_{D}(F) / T_{D}(F) ;$

$\mathrm{H}^{1}(G, E(F))=\operatorname{ker}\left(\varphi_{1} \mid E(F)\right) / \operatorname{im}\left(\varphi_{2} \mid E(F)\right)=E(F)[2] ;$

$\mathrm{H}^{1}\left(G, R_{D}(F)\right)=\operatorname{ker}\left(\varphi_{1} \mid R_{D}(F)\right) / \operatorname{im}\left(\varphi_{2} \mid R_{D}(F)\right)=R_{D}(F) / 2 R_{D}(F) ;$

$\mathrm{H}^{1}\left(G, T_{D}(F)\right)=\operatorname{ker}\left(\varphi_{1} \mid T_{D}(F)\right) / \operatorname{im}\left(\varphi_{2} \mid T_{D}(F)\right)=T_{D}(F) / 2 T_{D}(F) ;$

$\mathrm{H}^{1}(G, N(E(K)))=\operatorname{ker}\left(\varphi_{1} \mid N(E(K))\right) / \operatorname{im}\left(\varphi_{2} \mid N(E(K))\right)=N(E(K))[2]$. 
Theorem 1.5. The order of the group $\mathrm{H}^{1}(G, E(K))$ is

$$
\begin{aligned}
\sharp H^{1}(G, E(K)) & =\frac{2^{r_{D, F}} \cdot \sharp E(F)[2]}{\left(E(K): E(F)+R_{D}(F)\right)}=\frac{2^{r_{D, F}} \cdot \sharp E(F)[2]}{(N(E(K)): 2 E(F))} \\
& =2^{r_{D, F}-r_{F}} \cdot(E(F): N(E(K))) .
\end{aligned}
$$

Proof. Let $A=R_{D}(F), B=E(K)$ and $C=N(E(K))$, their corresponding Herbrand quotients are

$$
h(A)=h_{0}(A) / h_{1}(A), h(B)=h_{0}(B) / h_{1}(B), h(C)=h_{0}(C) / h_{1}(C)
$$

where $h_{m}(\cdot)$ is the order of $\widehat{\mathrm{H}}^{m}(G, \cdot)(m=0,1)$ (see $\left.[\mathrm{AW}, \mathrm{p} .109]\right)$. Since $2 E(F) \subset$ $N(E(K)) \subset E(F), \operatorname{rank} E_{D}(F)=\operatorname{rank} R_{D}(F)$ and $\operatorname{rank} N(E(K))=\operatorname{rank} E(F)$, by Lemma 1.2 and Proposition 1.4, we have

$$
\begin{aligned}
h\left(R_{D}(F)\right) & =\frac{\sharp\left(R_{D}(F)^{G} / \varphi_{1}\left(R_{D}(F)\right)\right)}{\sharp \mathrm{H}^{1}\left(G, R_{D}(F)\right)}=\frac{\sharp E(F)[2]}{\sharp\left(R_{D}(F) / 2 R_{D}(F)\right)}=2^{-r_{D}, F}, \\
h(E(K)) & =\frac{\sharp\left(E(K)^{G} / \varphi_{1}(E(K))\right)}{\sharp H^{1}(G, E(K))}=\frac{\sharp(E(F) / N(E(K)))}{\sharp H^{1}(G, E(K))} \\
& =\frac{2^{r_{F}} \cdot \sharp E(F)[2]}{\sharp \mathrm{H}^{1}(G, E(K)) \cdot(N(E(K)): 2 E(F))}, \\
h(N(E(K))) & =\frac{\sharp\left(N(E(K))^{G} / \varphi_{1}(N(E(K)))\right)}{\sharp \mathrm{H}^{1}(G, N(E(K)))}=\frac{\sharp(N(E(K)) / 2 N(E(K)))}{\sharp N(E(K))[2]}=2^{r_{F}} .
\end{aligned}
$$

Since $O \longrightarrow R_{D}(F) \longrightarrow E(K) \longrightarrow^{\varphi_{1}} N(E(K)) \longrightarrow O$ is an exact sequence of $G$-modules, by the theorem of Herbrand quotient (see [AW], Prop.10 on p.109), we have $h(E(K))=h\left(R_{D}(F)\right) \cdot h(N(E(K)))$. Therefore by the above calculation and Lemma 1.3, we get

$$
\begin{aligned}
\sharp \mathrm{H}^{1}(G, E(K)) & =\frac{2^{r_{D, F}} \cdot \sharp E(F)[2]}{(N(E(K)): 2 E(F))}=\frac{2^{r_{D, F}} \cdot \sharp E(F)[2]}{\left(E(K): E(F)+R_{D}(F)\right)} \\
& =2^{r_{D, F}-r_{F}} \cdot(E(F): N(E(K))) .
\end{aligned}
$$


Corollary 1.6. If $r_{F}=0$ and $E(F)[2]=\{O\}$, then $E(K)=E(F)+R_{D}(F)$ and $\sharp \mathrm{H}^{1}(G, E(K))=2^{r_{D, F}}=2^{r_{K}}$.

Proof. If $r_{F}=0$ and $E(F)[2]=\{O\}$, then by the Mordell-Weil theorem, $E(F) / 2 E(F) \cong(\mathbb{Z} / 2 \mathbb{Z})^{r_{F}} \oplus E(F)[2]=0$. So $(N(E(K)): 2 E(F))=1$ because $N(E(K)) / 2 E(F) \subset E(F) / 2 E(F)$, and then the conclusions follow from Lemma 1.3 and Theorem 1.5.

\section{A refined version of Yu's formula in the case of elliptic curves}

For the quadratic extension $K / F$ of number fields and the elliptic curve $E$ (over $F)$ as above, write $M_{F}\left(\right.$ resp. $\left.M_{K}\right)$ for a complete set of places on $F$ (resp. $K$ ), let $S_{\infty}$ be the set of infinite (i.e., Archimedean) places of $F$ and $S$ be the set of finite places of $F$ obtained by collecting together all places that ramify in $K / F$ and all places of bad reduction for $E / F$. Fix a place $w \in M_{K}$ lying above $v$ for each $v \in M_{F}$. Denote $\operatorname{Gal}\left(K_{w} / F_{v}\right)$ by $G_{w}$, where $F_{v}$ and $K_{w}$ are the completions of $F$ at $v$ and $K$ at $w$, respectively. For each real place $v \in S_{\infty}$, let $\sigma_{v}: F \rightarrow F_{v}=\mathbb{R}$ be the corresponding real embedding, so $\sigma_{v}(a) \in \mathbb{R}$ for any $a \in F$. For each finite place $v$ of $F$, we use $v(\cdot)$ to denote the normalized additive valuation of $F_{v}$, i.e., $v\left(F_{v}^{*}\right)=\mathbb{Z}$. Let $\|a\|_{F_{v}}=\left(\sharp k_{v}\right)^{-v(a)}\left(a \in F_{v}\right)$ denote the absolute value on $F_{v}\left(k_{v}\right.$ is the residue field of $F_{v}$ ), so is the meaning of $\|a\|_{K_{w}}$ on $K_{w}$. Let $\Delta_{v}, \Delta_{D, v}$, and $\Delta_{w}$ be the minimal discriminants for $E$ over $F_{v}, E_{D}$ over $F_{v}$ and $E$ over $K_{w}$ (see [Si1, p.186]), let $c_{v}, c_{D, v}$ and $c_{w}$ be the Tamagawa numbers for $E$ over $F_{v}, E_{D}$ over $F_{v}$ and $E$ over $K_{w}$ (see [Si1, p.451]), and let $d\left(K_{w} / F_{v}\right)$ be the discriminant of $K_{w} / F_{v}$, determined up to the square of a unit of $F_{v}$ (see $\left.[\mathrm{KT}, \mathrm{p} .332]\right)$. We also let $(,)_{F_{v}}$ denote the Hilbert 
norm-residue symbol, a bimultiplicative form $(,)_{F_{v}}: F_{v}^{*} \times F_{v}^{*} \rightarrow \mu_{2}=\{1,-1\}$ whose properties are described in [Se, Chapt.XIV]. For a vector space $V$ over $\mathbb{F}_{2}$, the finite field with 2 -elements, we denote its dimension by $\operatorname{dim}_{2} V$. Moreover, for $v \in M_{F} \backslash S_{\infty}$, we denote $\delta_{v}=\log _{2}\left(E\left(F_{v}\right): N\left(E\left(K_{w}\right)\right)\right)$. Then the Theorem 7.6 in [KT, p.332] states (in an equivalent form) that

$$
\delta_{v}=\log _{2}\left(\frac{c_{v} c_{D, v}}{c_{w}}\left(\frac{\left\|\Delta_{v} \Delta_{D, v} d\left(K_{w} / F_{v}\right)^{-6}\right\|_{F_{v}}}{\left\|\Delta_{w}\right\|_{K_{w}}}\right)^{1 / 12}\right) .
$$

Theorem 2.1 (A refined version of Yu's formula). Assume that the Shafarevich-Tate groups $\amalg(E / F), \amalg\left(E_{D} / F\right)$ and $\amalg(E / K)$ are finite. Then

$$
\frac{\sharp \amalg(E / F) \cdot \sharp \amalg\left(E_{D} / F\right)}{\sharp \amalg(E / K)}=2^{r_{D, F}-r_{F}-\delta(E, F, K)} \cdot(E(F): N(E(K)))^{2},
$$

where $\delta(E, F, K)=\delta_{\infty}+\delta_{f}$, with $\delta_{\infty}=\sharp\left\{v \in S_{\infty}: v\right.$ is ramified in $K$ and $\sigma_{v}(\Delta(E))>$ 0\} $(\Delta(E)$ is the discriminant of the elliptic curve $E$ over $F)$, and $\delta_{f}=\sum_{v \in S_{0}} \delta_{v}$. Here $S_{0}=\{v \in S: v$ is ramified or inertial in $K\}$.

Proof. By Yu's formula $[Y]$

$$
\frac{\sharp \amalg(E / F) \cdot \sharp \amalg\left(E_{D} / F\right)}{\sharp \amalg(E / K)}=\frac{\sharp \widehat{H}^{0}(G, E(K)) \cdot \sharp \mathrm{H}^{1}(G, E(K))}{\prod_{v \in M_{F}} \sharp \mathrm{H}^{1}\left(G_{w}, E\left(K_{w}\right)\right)} .
$$

By definition, $\widehat{\mathrm{H}}^{0}(G, E(K))=E(K)^{G} /(1+\sigma) E(K)=E(F) / N(E(K))$, so by the above Theorem 1.5, we get

$$
\frac{\sharp \amalg(E / F) \cdot \sharp \amalg\left(E_{D} / F\right)}{\sharp \amalg(E / K)}=\frac{2^{r_{D, F}-r_{F}} \cdot(E(F): N(E(K)))^{2}}{\prod_{v \in M_{F}} \sharp H^{1}\left(G_{w}, E\left(K_{w}\right)\right)} .
$$

On the other hand, by the Corollary 4.4 in [Ma, p.204], we have $\mathrm{H}^{1}\left(G_{w}, E\left(K_{w}\right)\right)=0$ for any $v \notin S \cup S_{\infty}$. Therefore

$$
\frac{\sharp \amalg(E / F) \cdot \sharp \amalg\left(E_{D} / F\right)}{\sharp \amalg(E / K)}=\frac{2^{r_{D, F}-r_{F}} \cdot(E(F): N(E(K)))^{2}}{\prod_{v \in S \cup S_{\infty}} \sharp \mathrm{H}^{1}\left(G_{w}, E\left(K_{w}\right)\right)} .
$$


By our assumption, the Shafarevich-Tate groups are finite; also $(E(F): N(E(K)))<$ $\infty$ because $\operatorname{rank} E(F)=\operatorname{rank} N(E(K))$, so by the above formula $(2.1), \mathrm{H}^{1}\left(G_{w}, E\left(K_{w}\right)\right)$ is a finite set for each $v \in S \cup S_{\infty}$.

Let $v \in S_{\infty}$, if $v$ is unramified in $K$, then $\mathrm{H}^{1}\left(G_{w}, E\left(K_{w}\right)\right)=0$ because $K_{w}=F_{v}=\mathbb{R}$ or $\mathbb{C}$. So we may assume that $v$ is ramified in $K$, then $F_{v}=\mathbb{R}$ and $K_{w}=\mathbb{C}$. By the Theorem 2.4 of Chapter V in [Si2], we have

$$
\mathrm{H}^{1}\left(G_{w}, E\left(K_{w}\right)\right)=\mathrm{H}^{1}(\operatorname{Gal}(\mathbb{C} / \mathbb{R}), E(\mathbb{C})) \cong\left\{\begin{array}{l}
0 \quad \text { if } \sigma_{v}(\Delta(E))<0, \\
\mathbb{Z} / 2 \mathbb{Z} \quad \text { if } \sigma_{v}(\Delta(E))>0 .
\end{array}\right.
$$

Hence

$$
\prod_{v \in S_{\infty}} \sharp \mathrm{H}^{1}\left(G_{w}, E\left(K_{w}\right)\right)=\sharp(\mathbb{Z} / 2 \mathbb{Z})^{\delta_{\infty}}=2^{\delta_{\infty}} .
$$

Let $v \in S$, if $v \notin S_{0}$, then $v$ splits completely in $K$, so $K_{w}=F_{v}$ and then $\mathrm{H}^{1}\left(G_{w}, E\left(K_{w}\right)\right)=0$. For $v \in S_{0}$, since $\mathrm{H}^{1}\left(G_{w}, E\left(K_{w}\right)\right)$ is finite as mentioned above, by Tate local duality [Ta1] (see also [Ma, Prop.4.2]), we have $\sharp \mathrm{H}^{1}\left(G_{w}, E\left(K_{w}\right)\right)=$ $\left(E\left(F_{v}\right): N\left(E\left(K_{w}\right)\right)\right)$. Hence by the Theorem 7.6 and the Remark in [KT, pp. 332, 333] (or by Prop.1 $\sim 5$ in $[\mathrm{Kr}]$ ), we get

$$
\prod_{v \in S} \sharp \mathrm{H}^{1}\left(G_{w}, E\left(K_{w}\right)\right)=\prod_{v \in S_{0}} \sharp \mathrm{H}^{1}\left(G_{w}, E\left(K_{w}\right)\right)=2^{\delta_{f}} .
$$

Substitute (2.2) and (2.3) into (2.1), we get

$$
\frac{\sharp \amalg(E / F) \cdot \sharp \amalg\left(E_{D} / F\right)}{\sharp \amalg \amalg(E / K)}=2^{r_{D, F}-r_{F}-\delta(E, F, K)} \cdot(E(F): N(E(K)))^{2} .
$$

The proof is completed.

Remark. By the results of Kramer on the local norm index in [Kr], one can calculate $\delta_{f}$ (hence $\delta(E, F, K)$ ) for most cases as follows: 
$\delta_{f}=\delta_{g}+\delta_{m}+\delta_{a}$, where $\delta_{g}, \delta_{m}$ and $\delta_{a}$ are defined as follows:

$$
\begin{aligned}
& \delta_{a}=\sum_{v \in S_{a}} \delta_{v} ; \\
& \delta_{m}=\delta_{s m r}+\delta_{n s m r} \text { with } \delta_{s m r}=\frac{1}{2} \sum_{v \in S_{s m r}}\left(1+\left(\Delta_{v}, D\right)_{F_{v}}\right) \text { and } \\
& \delta_{n s m r}=\frac{1}{2} \sum_{v \in S_{n s m r}^{\prime}}\left(1+(-1)^{v\left(\Delta_{v}\right)}\right)+\sum_{v \in S_{n s m r}^{\prime \prime}}\left(\frac{1}{2}\left(1+\left(\Delta_{v}, D\right)_{F_{v}}\right) \cdot(-1)^{v\left(\Delta_{v}\right)}+1\right) ; \\
& \delta_{g}=\sum_{v \in S_{g}} \operatorname{dim}_{2} \widetilde{E}_{v}\left(k_{v}\right)[2]+\sum_{v \in S_{g u}} \varepsilon(v), \quad \text { where } \\
& \varepsilon(v)= \begin{cases}\frac{1}{2}\left(1-(-1)^{v(D)}\right) \cdot\left[F_{v}: \mathbb{Q}_{2}\right] \text { if } E \text { has good supersingular reduction at } v, \\
\frac{1}{2}\left(3+\left(\Delta_{v}, D\right)_{F_{v}}\right) & \text { if } E \text { has good ordinary reduction at } v .\end{cases}
\end{aligned}
$$

Here $\widetilde{E_{v}}$ is the reduction of $E$ at $v, k_{v}$ is the residue field of $F_{v}$,

$S_{0}=\{v \in S: v$ is ramified or inertial in $K\}$;

$S_{g}=\left\{v \in S_{0}: v \nmid 2\right.$ and $E$ has good reduction at $\left.v\right\} ;$

$S_{g u}=\left\{v \in S_{0}: v \mid 2, E\right.$ has good reduction at $v$ and $F_{v}$ is unramified over $\left.\mathbb{Q}_{2}\right\}$;

$S_{a r}=\left\{v \in S_{0}: E\right.$ has additive reduction at $\left.v\right\}$;

$S_{a}=S_{a r} \cup\left\{v \in S_{0}: v \mid 2, E\right.$ has good reduction at $v$ and $F_{v}$ is ramified over $\left.\mathbb{Q}_{2}\right\}$;

$S_{s m r}=\left\{v \in S_{0}: E\right.$ has split multiplicative reduction at $\left.v\right\}$

$S_{n s m r}=\left\{v \in S_{0}: E\right.$ has non-split multiplicative reduction at $\left.v\right\}$

$=S_{n s m r}^{\prime} \sqcup S_{n s m r}^{\prime \prime}($ the disjoint union), where

$S_{n s m r}^{\prime}=\left\{v \in S_{n s m r}: v\right.$ is inertial in $\left.K\right\}$,

$S_{n s m r}^{\prime \prime}=\left\{v \in S_{n s m r}: v\right.$ is ramified in $\left.K\right\}$.

Obviously, $S_{0}=S_{g} \sqcup S_{g u} \sqcup S_{a} \sqcup S_{s m r} \sqcup S_{n s m r}$ (the disjoint union).

A Note Added. The main aim of this paper is to explicitly work out the orders of Shafarevich-Tate groups of some elliptic curves in quadratic number fields by refining Yu's formula. Nevertheless, as pointed out by the anonymous referee, 
the above methods can apply to more general cases. More precisely, as stated in the referee's comments, the above Theorem 1.5 holds verbatim for an abelian variety over a number field $F$ (or even a global field of characteristic different from 2), and, except for Lemma 1.1, the entire discussion can take place in the context of a group $G$ of order 2 acting linearly on a finitely generated abelian group $A$. Then the refined Yu's formula for an abelian variety $A / F$ is

$$
\frac{\sharp \amalg(A / F) \cdot \sharp \amalg\left(A_{D} / F\right)}{\sharp \amalg(A / K)}=\frac{2^{r_{D, F}-r_{F}} \cdot\left(A^{\prime}(F): N\left(A^{\prime}(K)\right)\right) \cdot(A(F): N(A(K)))}{\prod_{v}\left(A\left(F_{v}\right): N\left(A\left(K_{w}\right)\right)\right)},
$$

where $A^{\prime}$ is the dual abelian variety of $A$.

It would be interesting to find a similar formula of Kramer-Tunnell for higherdimensional abelian varieties to make these local norm cokernel quantities (i.e. $\left.\left(A\left(F_{v}\right): N\left(A\left(K_{w}\right)\right)\right)\right)$ explicitly calculated.

\section{Application I - congruent numbers elliptic curves}

Let $n \in \mathbb{Z} \backslash\{0,1\}$ be a square free integer and $K=\mathbb{Q}(\sqrt{n})$ be a quadratic number field. In this section, we consider elliptic curves $E: y^{2}=x^{3}-x$ and $E_{n}: y^{2}=x^{3}-n^{2} x$. All these curves have complex multiplication by $\mathbb{Z}[\sqrt{-1}]$, the Gaussian integral ring. Let $w \in M_{K}$ be a place of $K$ lying over 2 , as in section 2 above, recall that the notations $\Delta_{w}$ and $c_{w}$ represent the minimal discriminant and the Tamagawa factor for $E$ over $K_{w}$, respectively. Denote by $\operatorname{ord}_{w}$ the normalized additive valuation of $K_{w}$.

Lemma 3.1. We have

$$
\operatorname{ord}_{w}\left(\Delta_{w}\right)= \begin{cases}6 & \text { if } n \equiv 1(\bmod 4), \\ 12 & \text { if } n \equiv 2 \text { or } 3(\bmod 4), \quad \text { and }\end{cases}
$$




$$
c_{w}= \begin{cases}4 & \text { if } n \equiv 2 \text { or } 7(\bmod 8) \\ 2 & \text { if } n \equiv 1,3,5 \text { or } 6(\bmod 8)\end{cases}
$$

Proof. Up to isomorphisms, there are exactly seven quadratic extensions of $\mathbb{Q}_{2}$, namely, $\mathbb{Q}_{2}(\sqrt{-1}), \mathbb{Q}_{2}(\sqrt{-2}), \mathbb{Q}_{2}(\sqrt{2}), \mathbb{Q}_{2}(\sqrt{-3}), \mathbb{Q}_{2}(\sqrt{3}), \mathbb{Q}_{2}(\sqrt{-6}), \mathbb{Q}_{2}(\sqrt{6})$ (see [W, p.248]). Furthermore, one can easily verify that $K_{w} \cong \mathbb{Q}_{2} \Longleftrightarrow n \equiv 1(\bmod 8) ; \quad K_{w} \cong \mathbb{Q}_{2}(\sqrt{-3}) \Longleftrightarrow n \equiv 5(\bmod 8) ;$

$K_{w} \cong \mathbb{Q}_{2}(\sqrt{-1}) \Longleftrightarrow n \equiv 7(\bmod 8) ; \quad K_{w} \cong \mathbb{Q}_{2}(\sqrt{3}) \Longleftrightarrow n \equiv 3(\bmod 8) ;$

$K_{w} \cong \mathbb{Q}_{2}(\sqrt{-2}) \Longleftrightarrow n \equiv 14(\bmod 16) ; \quad K_{w} \cong \mathbb{Q}_{2}(\sqrt{2}) \Longleftrightarrow n \equiv 2(\bmod 16) ;$

$K_{w} \cong \mathbb{Q}_{2}(\sqrt{-6}) \Longleftrightarrow n \equiv 10,26$ or $42(\bmod 48) ;$

$K_{w} \cong \mathbb{Q}_{2}(\sqrt{6}) \Longleftrightarrow n \equiv 6,22$ or $38(\bmod 48)$

Next, by Tate's algorithm (see [Ta2, pp.47 52], [Si2, Chapt.IV§9]), we get

$$
\begin{gathered}
c_{w}=\left\{\begin{aligned}
2 & \text { if } K_{w}=\mathbb{Q}_{2}, \mathbb{Q}_{2}(\sqrt{-3}), \mathbb{Q}_{2}(\sqrt{-2}), \mathbb{Q}_{2}(\sqrt{3}) \text { or } \mathbb{Q}_{2}(\sqrt{6}), \\
4 & \text { if } K_{w}=\mathbb{Q}_{2}(\sqrt{-1}), \mathbb{Q}_{2}(\sqrt{2}) \text { or } \mathbb{Q}_{2}(\sqrt{-6}), \quad \text { and }
\end{aligned}\right. \\
\operatorname{ord}_{w}\left(\Delta_{w}\right)= \begin{cases}6 & \text { if } n \equiv 1(\bmod 4), \\
12 & \text { if } n \equiv 2 \text { or } 3(\bmod 4),\end{cases}
\end{gathered}
$$

from which the conclusion follows, and the proof is completed.

Lemma 3.2. We have

$$
\delta(E, \mathbb{Q}, K)= \begin{cases}2 \omega_{0}(n) & \text { if } n>0 \text { and } n \equiv 1(\bmod 8) \\ 1+2 \omega_{0}(n) & \text { if } n>0 \text { and } n \equiv 5 \text { or } 7(\bmod 8) \\ 3+2 \omega_{0}(n) & \text { if } n>0 \text { and } n \equiv 6(\bmod 8) \\ 2+2 \omega_{0}(n) & \text { if } n>0 \text { and } n \equiv 2 \text { or } 3(\bmod 8) \\ 1+2 \omega_{0}(n) & \text { if } n<0 \text { and } n \equiv 1(\bmod 8), \\ 2+2 \omega_{0}(n) & \text { if } n<0 \text { and } n \equiv 5 \text { or } 7(\bmod 8) \\ 3+2 \omega_{0}(n) & \text { if } n<0 \text { and } n \equiv 2 \text { or } 3(\bmod 8) \\ 4+2 \omega_{0}(n) & \text { if } n<0 \text { and } n \equiv 6(\bmod 8)\end{cases}
$$

where $\omega_{0}(n)$ is the number of odd prime divisors of $n$.

Proof. Since $\Delta(E)=64>0, E$ has good reduction everywhere except at 2 with additive reduction. So, by definition, $S=\{2\} \cup\{p: p$ is a prime and $p \mid$ 
$n\}, S_{g u}=S_{s m r}=S_{n s m r}=\emptyset$ and $S_{g}=S \backslash\{2\}$. So $\delta_{m}=0$, and $\delta_{\infty}=0$ (resp., 1 ) if $n>0$ (resp., $n<0$ ). Moreover, for each odd prime $p, E$ has good reduction at $p$, and it easy to see that $\widetilde{E}\left(\mathbb{F}_{p}\right)[2] \cong(\mathbb{Z} / 2 \mathbb{Z})^{2}$, so $\delta_{g}=\sum_{p \in S_{g}} \operatorname{dim}_{2} \widetilde{E}\left(\mathbb{F}_{p}\right)[2]=2 \omega_{0}(n)$. Hence by definition, $\delta(E, \mathbb{Q}, K)=\delta_{\infty}+\delta_{g}+\delta_{m}+\delta_{a}=2 \omega_{0}(n)+\delta_{\infty}+\delta_{a}$. We divide our discussion into the following cases.

Case A. $n \equiv 1(\bmod 8)$. Then 2 splits completely in $K$, and then $S_{a}=\emptyset$, so $\delta_{a}=0$, which implies $\delta(E, \mathbb{Q}, K)=2 \omega_{0}(n)\left(\right.$ resp., $\left.2 \omega_{0}(n)+1\right)$ if $n>0$ (resp., $\left.n<0\right)$.

Case B. $n \equiv 2,3,5,6$ or $7(\bmod 8)$. Then 2 is ramified or inertial in $K$, so $S_{a}=\{2\}$. Let $w \in M_{K}$ be the unique place in $K$ lying above 2 , then $K_{w}=\mathbb{Q}_{2}(\sqrt{n})$ is a quadratic extension over $\mathbb{Q}_{2}$. By Thm.7.6 in $[\mathrm{KT}]$, we get

$$
\delta_{a}=\delta_{2}=\log _{2}\left(E\left(\mathbb{Q}_{2}\right): N\left(E\left(K_{w}\right)\right)\right)=\log _{2}\left(\frac{c_{2} c_{n, 2}}{c_{w}}\left(\frac{\left\|\Delta_{2} \Delta_{n, v} d_{w}^{-6}\right\|_{\mathbb{Q}_{2}}}{\left\|\Delta_{w}\right\|_{K_{w}}}\right)^{1 / 12}\right) .
$$

Now we only need to compute all the values of $c_{2}, c_{n, 2}, c_{w}, \Delta_{2}, \Delta_{n, v}, \Delta_{w}$ and $d_{w}$. Firstly, by a method in ([KT], p.331)

$$
d_{w}=d\left(K_{w} / \mathbb{Q}_{2}\right)= \begin{cases}n & \text { if } n \equiv 5(\bmod 8) \\ 4 n & \text { if } n \equiv 2 \text { or } 3(\bmod 4) .\end{cases}
$$

Next, for the elliptic curves $E$ and $E_{n}$ over $\mathbb{Q}_{2}$, by Tate's algorithm (see [Ta2, pp.47 52], [Si2, Chapt.IV§9]), one can easily obtain that $v_{2}\left(\Delta_{2}\right)=6, c_{2}=2$ and $v_{2}\left(\Delta_{n, 2}\right)=6$ if $n \equiv 3,5$ or $7(\bmod 8) ; \quad v_{2}\left(\Delta_{n, 2}\right)=12$ if $n \equiv 2$ or $6(\bmod 8) ;$ $c_{n, 2}=2$ if $n \equiv 3,5$ or $7(\bmod 8) ; \quad c_{n, 2}=4$ if $n \equiv 2$ or $6(\bmod 8)$. Also by Lemma 3.1 above, we have $\operatorname{ord}_{w}\left(\Delta_{w}\right)=12$ if $n \equiv 2$ or $3(\bmod 4) ; \quad \operatorname{ord}_{w}\left(\Delta_{w}\right)=6$ if $n \equiv 5(\bmod 8) ;$ $c_{w}=2$ if $n \equiv 3,5$ or $6(\bmod 8) ; \quad c_{w}=4$ if $n \equiv 2$ or $7(\bmod 8)$. 
Now substitute all of them into (3.1), the conclusion for case B then follows, and the proof is completed.

It is well known that the $L$ - function $L(E / \mathbb{Q}, s)=\sum b_{m} m^{-s}$ of the elliptic curve $E=E_{1}: y^{2}=x^{3}-x$ corresponds to a weight two cusp form $g=\sum b_{m} q^{m} \in$ $S_{2}\left(\Gamma_{0}(32)\right)$ (see [Kob, p.217]), and for the elliptic curve $E_{n}: y^{2}=x^{3}-n^{2} x$, by Tunnell's theorem (see [T, p.328] or [Kob, p.217]), there exist a form $f=\sum a_{m} q^{m} \in$ $S_{3 / 2}\left(\widetilde{\Gamma}_{0}(128)\right)$ and a form $f^{\prime}=\sum a_{m}^{\prime} q^{m} \in S_{3 / 2}\left(\widetilde{\Gamma}_{0}(128), \chi_{2}\right)$ such that their Shimura $\operatorname{lifts} \operatorname{Shimura}(f)=\operatorname{Shimura}\left(f^{\prime}\right)=g$ and

$$
L\left(E_{n} / \mathbb{Q}, 1\right)=\left\{\begin{array}{l}
\frac{\omega}{4 \sqrt{n}} a_{n}^{2} \text { if } n \text { is odd, } \\
\frac{\omega}{2 \sqrt{n}}\left(a_{n / 2}^{\prime}\right)^{2} \text { if } n \text { is even. }
\end{array}\right.
$$

where $\omega=\int_{1}^{\infty} \frac{d x}{\sqrt{x^{3}-x}}=2.6220575$ is the least positive period of $E / \mathbb{Q}$.

Theorem 3.3. Let $n$ be a square free integer satisfying one of the following conditions

(1) $n>0$ and $n \equiv 1,2$ or $3(\bmod 8)$;

(2) $n<0$ and $n \equiv 5,6$ or $7(\bmod 8)$.

Then for the elliptic curves $E_{n}: y^{2}=x^{3}-n^{2} x$ and $E=E_{1}$ as above, if the full BSD conjecture (see $\left[\operatorname{Si1}\right.$, p.452]) is true for $E_{n}$ over $\mathbb{Q}$ with $L\left(E_{n} / \mathbb{Q}, 1\right) \neq 0$, and $\amalg(E / \mathbb{Q}(\sqrt{n}))$ is finite, we have

$$
\sharp \amalg(E / \mathbb{Q}(\sqrt{n}))= \begin{cases}2^{-4} \cdot a_{n}^{2} & \text { if } n>0 \text { and } n \equiv 1(\bmod 8), \\ 2^{-2} \cdot a_{n}^{2} & \text { if } n>0 \text { and } n \equiv 3(\bmod 8), \\ 2^{-2} \cdot\left(a_{n / 2}^{\prime}\right)^{2} & \text { if } n>0 \text { and } n \equiv 2(\bmod 8), \\ 2^{-2} \cdot a_{-n}^{2} & \text { if } n<0 \text { and } n \equiv 5 \text { or } 7(\bmod 8), \\ \left(a_{-n / 2}^{\prime}\right)^{2} & \text { if } n<0 \text { and } n \equiv 6(\bmod 8),\end{cases}
$$

where $a_{|n|}$ and $a_{|n / 2|}^{\prime}$ are the Fourier coefficients of the above modular forms $f$ and $f^{\prime}$. 
Proof. We prove the case that $n>0$ satisfying $n \equiv 1(\bmod 8)$, the other cases can be similarly verified. For this case, by Lemma 3.2 above, $\delta(E, \mathbb{Q}, K)=2 \omega_{0}(n)$. By the assumption, $L\left(E_{n} / \mathbb{Q}, 1\right) \neq 0$ and the full BSD conjecture is true for $E_{n}$ over $\mathbb{Q}$, so $r_{n, \mathbb{Q}}=0$, IUI $\left(E_{n} / \mathbb{Q}\right)$ is finite and $L\left(E_{n} / \mathbb{Q}, 1\right) / \Omega_{E_{n} / \mathbb{Q}}=(\mathrm{BSD})_{\infty, \mathbb{Q}}\left(E_{n}\right)$ with $\Omega_{E_{n} / \mathbb{Q}}=\omega / \sqrt{n}$, where

$$
(\mathrm{BSD})_{\infty, \mathbb{Q}}\left(E_{n}\right)=\operatorname{Reg}_{\infty, \mathbb{Q}}\left(E_{n}\right) \times \frac{\sharp \amalg\left(E_{n} / \mathbb{Q}\right) \prod_{v \in M_{\mathbb{Q}}} c_{v}}{\sqrt{d(\mathbb{Q})} \times \sharp E_{n}(\mathbb{Q})_{\text {tors }}^{2}} .
$$

We have $\operatorname{Reg}_{\infty, \mathbb{Q}}\left(E_{n}\right)=1$ because $r_{n, \mathbb{Q}}=0$; obviously, $d(\mathbb{Q})=1$; also $\sharp E_{n}(\mathbb{Q})_{\text {tors }}=4$ (see [Si1], pp.346, 347); moreover, $c_{\infty}=2$ because $E_{n}$ is not connected over $\mathbb{R}$, then from [R2, p.235] we have

$$
\begin{aligned}
& \prod_{v \in M_{\mathbb{Q}}} c_{v}=c_{\infty} \cdot \prod_{p<\infty} c_{p}=2 \times 2^{2 \omega_{0}(n)+1}=2^{2 \omega_{0}(n)+2}, \text { hence } \\
& L\left(E_{n} / \mathbb{Q}, 1\right)=\Omega_{E_{n} / \mathbb{Q}} \times(\mathrm{BSD})_{\infty, \mathbb{Q}}\left(E_{n}\right)=2^{2 \omega_{0}(n)-2} \times \frac{\omega}{\sqrt{n}} \times \sharp \amalg\left(E_{n} / \mathbb{Q}\right) .
\end{aligned}
$$

On the other hand, by Tunnell's theorem (see [T, Thm.3, p.328] or [Kob, p.217]), we have $L\left(E_{n} / \mathbb{Q}, 1\right)=\omega a_{n}^{2} /(4 \sqrt{n})$ with the Fourier coefficient $a_{n}$ of the modular form $f$ mentioned above. Therefore by $(3.2)$, we get $\sharp \amalg\left(E_{n} / \mathbb{Q}\right)=2^{-2 \omega_{0}(n)} \cdot a_{n}^{2}$. As mentioned before, $\amalg \amalg \amalg(E / \mathbb{Q})=0$, and $E(\mathbb{Q})=E(\mathbb{Q})[2] \cong(\mathbb{Z} / 2 \mathbb{Z})^{2}$, so $r_{K}=$ $r_{n, \mathbb{Q}}+r_{\mathbb{Q}}=0$, and it is easy to know that $E(K)_{\text {tors }}=E(\mathbb{Q})[2]$, hence by definition, we have $(E(\mathbb{Q}): N(E(K)))=4$. By assumption, $\amalg(E / \mathbb{Q}(\sqrt{n}))$ is finite, hence by the above refined Yu's formula, we get

$$
\begin{aligned}
\sharp \amalg(E / \mathbb{Q}(\sqrt{n})) & =2^{-r_{n, \mathbb{Q}}+r_{\mathbb{Q}}+\delta(E, \mathbb{Q}, K)} \cdot(E(\mathbb{Q}): N(E(K)))^{-2} \cdot \sharp \amalg(E / \mathbb{Q}) \cdot \sharp \amalg\left(E_{n} / \mathbb{Q}\right) \\
& =2^{2 \omega_{0}(n)} \cdot 4^{-2} \cdot 2^{-2 \omega_{0}(n)} \cdot a_{n}^{2}=2^{-4} a_{n}^{2} . \quad \text { In particular, } \\
& \frac{\sharp \amalg(E / \mathbb{Q}(\sqrt{n}))}{\sharp \amalg\left(E_{n} / \mathbb{Q}\right)}=2^{2 \omega_{0}(n)-4} .
\end{aligned}
$$


Therefore the conclusion of the Shafarevich-Tate groups $\amalg(E / \mathbb{Q}(\sqrt{n}))$ is obtained, and the proof of is completed.

Remark. (1) Note that $E$ and $E_{n}$ are isomorphic over $\mathbb{Q}(\sqrt{n})$, and $E_{n}=E_{-n}$, so in particular $\sharp \amalg\left(E_{n} / \mathbb{Q}(\sqrt{ \pm n})\right)=\sharp \amalg \amalg(E / \mathbb{Q}(\sqrt{ \pm n}))$, and one has all the same results for $E_{n}$ over $\mathbb{Q}(\sqrt{n})$ as $E$ in the above Theorem 3.3. Moreover, the Fourier coefficients $a_{n}$ and $a_{n}^{\prime}(n>0)$ can be determined by the number of solutions of some concrete quadratic forms in three variables (see Tunnell's theorem in [T,p.323 and p.325] for the detail).

(2) The above proof of Theorem 3.3 also shows that the ratio $\sharp \amalg(E / \mathbb{Q}(\sqrt{n})) / \sharp \amalg\left(E_{n} / \mathbb{Q}\right)$ can be arbitrarily large. For example, for a square free positive integer $n$ satisfying the condition of the following Corollary 3.4.(3), then $\sharp \amalg(E / \mathbb{Q}(\sqrt{n})) / \sharp \amalg\left(E_{n} / \mathbb{Q}\right)=\left(2^{-2} \cdot a_{n}^{2}\right) /\left(2^{-2 \omega_{0}(n)} \cdot a_{n}^{2}\right)=2^{2 \omega_{0}(n)-2}$, where $\omega_{0}(n)$ is the number of odd prime divisors of $n$.

Corollary 3.4. For the elliptic curves $E_{n}: y^{2}=x^{3}-n^{2} x$ and $E=E_{1}$ as above, assume that $\amalg(E / \mathbb{Q}(\sqrt{n}))$ is finite.

(1) If $n= \pm p, p$ is a prime number, and $p \equiv 3(\bmod 8)$, then

$$
\sharp \amalg(E / \mathbb{Q}(\sqrt{p}))=\sharp \amalg(E / \mathbb{Q}(\sqrt{-p}))=\frac{1}{4} a_{p}^{2},
$$

in particular, all such $a_{p}$ are even.

(2) Suppose $n= \pm p_{1} \cdots p_{m} \equiv 1(\bmod 4)$, where $p_{1}, \cdots, p_{m}$ are distinct prime numbers with $p_{i} \not \equiv 5(\bmod 8)$. If $s_{k}(n)=1$, then

$$
\sharp \amalg(E / \mathbb{Q}(\sqrt{n}))= \begin{cases}2^{-4} \cdot a_{n}^{2} & \text { if } n>0 \text { and } n \equiv 1(\bmod 8), \\ 2^{-2} \cdot a_{-n}^{2} & \text { if } n<0 \text { and } n \equiv 5(\bmod 8) .\end{cases}
$$

(3) Suppose $n=p_{1} \cdots p_{m}$, where $p_{1}, \cdots, p_{m}$ are distinct prime numbers with $p_{1} \equiv$ 
$3(\bmod 8)$ and $p_{2} \equiv \cdots \equiv p_{m} \equiv 1(\bmod 8)$. If $s_{2 m-1}(-n)=1$, then

$$
\sharp \amalg(E / \mathbb{Q}(\sqrt{n}))=2^{-2} \cdot a_{n}^{2} \text {, in particular, } 2^{m} \| a_{n} \text {, i.e., } v_{2}\left(a_{n}\right)=m \text {. }
$$

Here $s_{k}(n)$ and $s_{2 m-1}(-n)$ are the $\mathbb{F}_{2}-$ valued functions on $n$ and its Gaussian prime factors defined in [Z, p.387].

Proof. (1). By a theorem of Rubin (see [R1], P.26), the full BSD conjecture is true for $E_{p}: y^{2}=x^{3}-p^{2} x$ over $\mathbb{Q}$ and $L\left(E_{p} / \mathbb{Q}, 1\right) \neq 0$, so the conclusion follows directly from the above Theorem 3.3.

(2) and (3). By the Theorem 2 and Proposition 3 in [Z, p.387], the full BSD conjecture is true for $E_{n}: y^{2}=x^{3}-n^{2} x$ over $\mathbb{Q}$ and $L\left(E_{n} / \mathbb{Q}, 1\right) \neq 0$ in these cases, so the conclusion of the orders of the Shafarevich-Tate groups for $E$ over $\mathbb{Q}(\sqrt{n})$ follows directly from the above Theorem 3.3. Now we come to compute the 2 -adic valuation of $a_{n}$ in case (3). In fact, by the Theorem 2 in [Z, p.387], we know that, if $s_{2 m-1}(-n)=1$, then $L\left(E_{n} / \mathbb{Q}, 1\right) \neq 0$ and the 2-Selmer group $S^{(2)}\left(E_{n} / \mathbb{Q}\right)$ has order 4. So by a theorem of Coates-Wiles (see [CW, Thm.1, p.223]), one has $r_{n, \mathbb{Q}}=\operatorname{rank} E_{n}(\mathbb{Q})=0$, so $E_{n}(\mathbb{Q}) / 2 E_{n}(\mathbb{Q}) \cong E_{n}(\mathbb{Q})[2] \cong(\mathbb{Z} / 2 \mathbb{Z})^{2}$. Then by the exact sequence (see [Si1], chapt.X, Thm.4.2)

$$
0 \rightarrow E_{n}(\mathbb{Q}) / 2 E_{n}(\mathbb{Q}) \rightarrow S^{(2)}\left(E_{n} / \mathbb{Q}\right) \rightarrow \amalg\left(E_{n} / \mathbb{Q}\right)[2] \rightarrow 0
$$

we get $\mathbb{I} \amalg\left(E_{n} / \mathbb{Q}\right)[2]=0$, hence the 2 -primary part $\mathbb{I} \amalg\left(E_{n} / \mathbb{Q}\right)\left[2^{\infty}\right]=0$, and so $\sharp \amalg\left(E_{n} / \mathbb{Q}\right)$ is odd. But, from the fact that the full BSD conjecture for $E_{n}$ over $\mathbb{Q}$, it is easy to know that $\sharp \amalg\left(E_{n} / \mathbb{Q}\right)=2^{-2 m} a_{n}^{2}$ (see the above proof of Theorem 3.3), so $v_{2}\left(a_{n}\right)=m$. The proof is completed.

\section{Application II - Elliptic curves related with Heegner points.}


In this section, let $E$ be an elliptic curve defined over $\mathbb{Q}, N_{E}$ be the conductor of $E / \mathbb{Q}$, let $K=\mathbb{Q}(\sqrt{D})$ be an imaginary quadratic field with fundamental discriminant $D$ satisfying the Heegner hypothesis, that is,

Heegner hypothesis. All prime numbers $p$ dividing $N_{E}$ are split in $K$.

Then there exists a Heegner point $P_{K} \in E(K)$ (see [GZ], [Kol1 3]). We have the following results of Shafarevich-Tate groups and Heegner points:

Theorem 4.1. (1) Let $E$ be an elliptic curve defined over $\mathbb{Q}$, and $K=\mathbb{Q}(\sqrt{D})$ be an imaginary quadratic field satisfying the Heegner hypothesis. Let $P_{K}$ be a Heegner point of $E(K)$, if $P_{K}$ is of infinite order, then $\frac{\sharp \amalg(E / \mathbb{Q}) \cdot \sharp \amalg\left(E_{D} / \mathbb{Q}\right)}{\sharp \amalg(E / K)}=\left\{\begin{array}{l}2^{1-\delta_{\infty}-\delta_{g}} \cdot(E(\mathbb{Q}): N(E(K)))^{2} \quad \text { if } L(E / \mathbb{Q}, 1) \neq 0, \\ 2^{-1-\delta_{\infty}-\delta_{g}} \cdot(E(\mathbb{Q}): N(E(K)))^{2} \quad \text { if } L(E / \mathbb{Q}, 1)=0 .\end{array}\right.$

(2) For the elliptic curve $E: y^{2}=x^{3}-x+\frac{1}{4}$ and the imaginary quadratic field $K=\mathbb{Q}(\sqrt{D})$ satisfying the Heegner hypothesis, if the Heegner point $P_{K} \in E(K)$ is of infinite order, then

$$
\sharp \amalg(E / K)=2^{\delta_{g}} \cdot \sharp \amalg\left(E_{D} / \mathbb{Q}\right) .
$$

In particular, for each $D \in\{-7,-11,-47,-71,-83,-84,-127,-159,-164,-219$, $-231,-263,-271,-287,-292,-303,-308,-359,-371,-404,-443,-447,-471\}$, the group $\amalg(E / K)$ is trivial.

Proof. (1) For the elliptic curve $E / \mathbb{Q}$ and the field $K$, by definition, $S=\{p$ : $p$ is a prime number and $\left.p \mid D N_{E}\right\}$. By the Heegner hypothesis, $N_{E}$ is prime to $D$, and $S_{0}=\{p: p$ is a prime number and $p \mid D\}$, in particularly, $E$ has good reduction at each prime $p \in S_{0}$, so $S_{g} \cup S_{g u}=S_{0}$, and then $S_{a}=S_{s m r}=S_{n s m r}=\emptyset$. Hence by definition, $\delta(E, \mathbb{Q}, K)=\delta_{\infty}+\delta_{g}$ with $\delta_{\infty}=1($ resp., 0$)$ if $\Delta(E)>0($ resp. $\Delta(E)<0)$. 
On the other hand, by the Heegner hypothesis, from the functional equation we have $L(E / K, 1)=0$. Since the Heegner point $P_{K}$ is of infinite order, by the formula of Gross-Zagier (see [GZ, p.311]), the analytic $\operatorname{rank} \operatorname{ord}_{s=1} L(E / K, s)=1$, which implies

$$
\begin{aligned}
& \operatorname{ord}_{s=1} L(E / \mathbb{Q}, s)=1 \quad \text { and } \quad L\left(E_{D} / \mathbb{Q}, 1\right) \neq 0 ; \quad \text { or } \\
& \operatorname{ord}_{s=1} L\left(E_{D} / \mathbb{Q}, s\right)=1 \quad \text { and } \quad L(E / \mathbb{Q}, 1) \neq 0 .
\end{aligned}
$$

Then by the theorems of Kolyvagin and Gross-Zagier (see [Kol1 3], [GZ]), we know that $r_{\mathbb{Q}}=\operatorname{ord}_{s=1} L(E / \mathbb{Q}, s)$ and $r_{D, \mathbb{Q}}=\operatorname{ord}_{s=1} L\left(E_{D} / \mathbb{Q}, s\right)$, moreover, all the groups $\amalg \amalg(E / K), \amalg \amalg(E / \mathbb{Q})$ and $\amalg \amalg\left(E_{D} / \mathbb{Q}\right)$ are finite. The conclusion then follows from the above refined Yu's formula. This proves (1).

(2) For the elliptic curve $E: y^{2}=x^{3}-x+\frac{1}{4}$, its discriminant $\Delta(E)=N_{E}=37>0$, and the equation $y^{2}+y=x^{3}-x$ is a global minimal equation of $E$ over $\mathbb{Q}$. By a theorem of Kolyvagin (see $[\mathrm{Kol} 3, \mathrm{p} .444])$, we know that $L(E / \mathbb{Q}, 1)=0, r_{\mathbb{Q}}=1$ and $\amalg(E / \mathbb{Q})=0$, moreover, $E(\mathbb{Q})=\mathbb{Z} P_{0}$ with $P_{0}=\left(0, \frac{1}{2}\right)$. Now from the proof of $(1)$, we have $\delta_{\infty}=1$ because $\Delta(E)>0$, then by the formula in (1), we get

$$
\sharp \amalg(E / K)=2^{2+\delta_{g}} \cdot(E(\mathbb{Q}): N(E(K)))^{-2} \cdot \sharp \amalg\left(E_{D} / \mathbb{Q}\right) .
$$

Since $E(\mathbb{Q}) / 2 E(\mathbb{Q}) \cong \mathbb{Z} / 2 \mathbb{Z} \times E(\mathbb{Q})[2]=\mathbb{Z} / 2 \mathbb{Z}$ because $E(\mathbb{Q})[2]=0$, by definition, $(E(\mathbb{Q}): N(E(K))) \mid(E(\mathbb{Q}): 2 E(\mathbb{Q}))=2$, hence $N(E(K))=E(\mathbb{Q})$ or $2 E(\mathbb{Q})$. But by the group law algorithm (see [Si1, p.53]), it is not difficult to verify that $P_{0} \notin N(E(K))$, which implies $N(E(K))=2 E(\mathbb{Q})$, so $(E(\mathbb{Q}): N(E(K)))=2$. Substituting it into (4.1), we get

$$
\sharp \amalg(E / K)=2^{\delta_{g}} \cdot \sharp \amalg\left(E_{D} / \mathbb{Q}\right) .
$$


This proves the first conclusion in (2).

Now we assume that $D$ is one of the given 23 integers. Then by a theorem of Kolyvagin $\left(\right.$ see $[\mathrm{Kol} 2]$, p.477), $\amalg\left(E_{D} / \mathbb{Q}\right)=0$. So we only need to compute $\delta_{g}$. From the discussion in (1), we know that $S_{g} \cup S_{g u}=S_{0}$, moreover, it is easy to know that $S_{g u}=\{2\}$ if and only if $D$ is even, otherwise, $S_{g u}=\emptyset$. Furthermore, it can be seen easily that $E$ has good supersingular reduction at 2 . Hence by definition, we have

$$
\delta_{g}=\sum_{p \in S_{0} \backslash\{2\}} \operatorname{dim}_{2} \widetilde{E_{p}}\left(\mathbb{F}_{p}\right)[2]+\varepsilon(2)
$$

with $\varepsilon(2)=\frac{1}{2}\left(1-(-1)^{v_{2}(D)}\right)$ (resp., 0$)$ if $D$ is even (resp., odd). Obviously, $\varepsilon(2)=0$ for each of these 23 integers, and by calculation, it can be easily seen that $\widetilde{E_{p}}\left(\mathbb{F}_{p}\right)[2]=$ $\{O\}$ for each $p \in S_{0} \backslash\{2\}$, which implies $\delta_{g}=0$. Therefore, by (4.2), we get $\sharp \amalg(E / K)=\sharp \amalg \amalg\left(E_{D} / \mathbb{Q}\right)=1$, that is, $\amalg \amalg(E / K)$ is trivial. This proves (2), and the proof of is completed.

By the methods of this paper, one can obtain other similar examples as done in Corollary 3.4 and Theorem 4.1 above.

Acknowledgments. I would like to thank the anonymous referee for a very careful reading of the paper and many helpful comments and suggestions.

\section{References}

[ABF ] J. Antoniadis, M. Bungert, G. Frey, Properties of twists of elliptic curves, J. reine angew. math. 405 (1990), 1-28.

[AW ] M. Atiyah, C.T.C. Wall, Cohomology of groups, in: Algebraic Number Theory (J.W.S. Cassels and A. Frohlich, Eds.), pp.94-115, London: Academic Press, 1967. 
[CW ] J. Coates, A. Wiles, On the conjecture of Birch and Swinnerton-Dyer, Invent. math., 39 (1977), 223-251.

[GZ ] B.H. Gross, D.B. Zagier, Heegner points and derivatives of $L$-series, Invent. math., 84 (1986), 225-320.

[Kob ] N. Koblitz, Introduction to Elliptic Curves and Modular Forms, 2nd Edition, New York: Springer-Verlag, 1993.

[Kol1 ] V.A. Kolyvagin, Finiteness of $E(\mathbb{Q})$ and $\amalg \amalg(E / \mathbb{Q})$ for a subclass of Weil curves, (Russian) Izv. Akad. Nauk SSSR Ser. Mat. 52 (1988), 522-540, 670-671; translation in Math. USSR-Izv. 32 (1989), 523-541.

[Kol2 ] V.A. Kolyvagin, The Mordell-Weil and Shafarevich-Tate groups for Weil elliptic curves, (Russian) Izv. Akad. Nauk SSSR Ser. Mat. 52 (1988), 11541180, 1327; translation in Math. USSR-Izv. 33 (1989), 473-499.

[Kol3 ] V.A. Kolyvagin, Euler systems. In The Grothendieck Festschrift, Vol. II, 435-483, Progr. Math. 87, Birkhauser Boston, Boston, MA, 1990.

[Kr ] K. Kramer, Arithmetic of elliptic curves upon quadratic extension, Transactions of the American Mathematical Society, 264 (1981), 121-135.

[KT ] K. Kramer, J. Tunnell, Elliptic curves and local $\varepsilon$-factors, Compositio Math., 46 (1982), 307-352.

[Ma ] B. Mazur, Rational points of Abelian varieties with values in towers of number fields, Invent. math., 18 (1972), 183-266. 
[R1 ] K. Rubin, The main conjectures of Iwasawa theory for imaginary quadratic fields, Invent. math., 103 (1991), 25-68.

[R2 ] K. Rubin, Fudge factors in the Birch and Swinnerton-Dyer conjecture, in: Ranks of Elliptic Curves and Random Matrix Theory (J.B. Conrey, D.W. Farmer, F. Mezzadri and N.C. Snaith Eds.), pp.233-236, Cambridge: Cambridge University Press, 2007.

[RS ] K. Rubin, A. Silverberg, Rank frequencies for quadratic twists of elliptic curves, Experiment Math., 10 (2001), 559-569.

[Se ] J. -P. Serre, Local Fields, New York: Springer-Verlag, 1979.

[Si1 ] J. H. Silverman, The Arithmetic of Elliptic Curves, GTM 106, 2nd Edition, New York: Springer-Verlag, 2009.

[Si2 ] J. H. Silverman, Advanced topics in the Arithmetic of Elliptic Curves, GTM 151, New York: Springer-Verlag, 1999.

[Ta1 ] J. Tate, Duality theorems in Galois cohomology over number fields. Proc. Intern. Congress Math. at Stockholm, 1962, 288-295. Institute Mittag-Leffler Djursholm, Sweden, 1963

[Ta2 ] J. Tate, Algorithm for determing the type of a singular fiber in an elliptic pencil, in: Modular functions of one variable, IV, (Proc. Internat. Summer School, Univ. Antwerp 1972), pp.33-52. Lecture Notes in Math. 476, Springer, Berlin, 1975. 
[T ] J. Tunnell, A classical Diophantine problem and modular forms of weight 3/2, Invent. math., 72 (1983), 323-334.

[W ] E. Weiss, Algebraic Number Theory, New York: McGraw-Hill Book C ompany, Inc, 1963.

[Y ] H. Yu, On Tate-Shafarevich groups over Galois extensions, Israel J. Math., 141 (2004), 211-220.

[Z ] C. Zhao, A criterion for elliptic curves with lowest 2-power in L(1), Math. Proc. Cambridge Philos. Soc. 121(1997), 385-400. 